\title{
On finite projective planes in Lenz-Barlotti class at least I.3
}

\author{
Dina Ghinelli and Dieter Jungnickel
}

To Professor Adriano Barlotti on the occasion of his 80th birthday

\begin{abstract}
We establish the connections between finite projective planes admitting a collineation group of Lenz-Barlotti type I.3 or I.4, partially transitive planes of type (3) in the sense of Hughes, and planes admitting a quasiregular collineation group of type $(\mathrm{g})$ in the DembowskiPiper classification; our main tool is an equivalent description by a certain type of difference set relative to disjoint subgroups which we will call a neo-difference set. We then discuss geometric properties and restrictions for the existence of planes of Lenz-Barlotti class I.4. As a side result, we also obtain a new synthetic description of projective triangles in desarguesian planes.
\end{abstract}

\section{Introduction}

We shall assume that the reader is familiar with the basic theory of finite projective planes, in particular with the notions of elations, homologies, $(p, L)$-transitivity and the idea of the Lenz-Barlotti classification. For background, we refer the reader to Dembowski [5], Hughes and Piper [15] or Pickert [23].

We shall be concerned with a couple of closely interrelated concepts which have appeared in various places in the literature:

- finite projective planes admitting a collineation group of Lenz-Barlotti type I.3 or I.4;

- partially transitive planes of type (3) in the sense of Hughes [14];

- planes admitting a quasiregular collineation group of type $(\mathrm{g})$ in the DembowskiPiper classification [6];

- a certain type of difference set relative to disjoint subgroups in the sense of Hiramine [10] which we will call a "neo-difference set", as the abelian case corresponds to neofields.

If one looks at the literature, some confusion is bound to arise, as the connections between these notions have not been made really precise. Our first aim is to clarify these connections. In particular, we establish that groups of Lenz-Barlotti type I.4 are equivalent to (necessarily abelian) quasiregular groups of type $(\mathrm{g})$; though this equivalence has been around in a vague way, it has never been proved in the litera- 
ture, and the proof is indeed not at all obvious. Such groups are also equivalent to abelian neo-difference sets; unfortunately, the only known examples occur in the desarguesian planes. Similarly, groups of Lenz-Barlotti type I.3 are equivalent to non-abelian neo-difference sets, and the only known examples come from nearfield planes.

Once the basic equivalences have been sorted out, we prove several known and also a few new restrictions for planes of Lenz-Barlotti class I.4, using the setting of abelian neo-difference sets. This allows us not only to avoid neofields for the major part of our exposition (using the standard machinery of group rings instead), but also to give simpler and more transparent proofs in many cases, stressing the analogy to planar and affine difference sets. In particular, we shall provide a short and transparent proof of the multiplier theorem for neo-difference sets.

We conclude this introduction with a little more background and a few more references. Recall that a permutation group $G$ is called quasiregular if it induces a regular action on each orbit: each group element fixes either none or all elements in the orbit. This condition is satisfied in particular when $G$ is abelian; more generally, it is easily seen that $G$ acts quasiregularly on a set if and only if every stabilizer is a normal subgroup.

The famous Lenz-Barlotti classification is due to [1], [19]; see Yaqub [28] for an old survey which is still worth reading. An up-to-date account of the DembowskiPiper classification [6] is given by the present authors [7]. Background on difference sets and group rings can be found in Chapter VI of Beth, Jungnickel and Lenz [2].

\section{Groups of type at least I.3}

In the Lenz-Barlotti classification, collineation groups of projective planes are classified according to the configuration $F$ formed by the point-line pairs $(p, L)$ for which the given group $G$ is $(p, L)$-transitive; in the special case $G=$ Aut $\Pi$, one speaks of the Lenz-Barlotti class of $\Pi$. For a group of type I.4, $F$ consists of the vertices and the opposite sides of a triangle; for type I.3, one of these transitivities is missing.

We begin by considering groups of type at least I.3. Thus we assume that $\Pi$ is a finite projective plane of order $n$ which is both $(y, o x)$ - and $(x, o y)$-transitive, where $o$, $x$, and $y$ form a triangle. We may think of $L_{\infty}=x y$ as the line at infinity, of $o$ as the origin, and of $o x$ and $o y$ as the $x$ - and $y$-axis, respectively. Points which are not on a side of the triangle oxy will be called ordinary points; ordinary lines are defined dually. We denote the group of all $(x, o y)$-homologies by $X$ and the group of all $(y, o x)$ homologies by $Y$; w.l.o.g., we may assume that the group $G$ under consideration is the group generated by $X$ and $Y$. We also fix an arbitrary ordinary point $u$ for the rest of our discussion. The following basic result is easy to prove, cf. Figure 1, and will be left to the reader. ${ }^{1}$

Lemma 2.1. With the notation above, $G$ is the direct product of $X$ and $Y$; moreover, $G$ acts regularly both on the set of ordinary points and on the set of ordinary lines.

\footnotetext{
${ }^{1}$ For a detailed treatment including more background and complete proofs, see [8].
} 


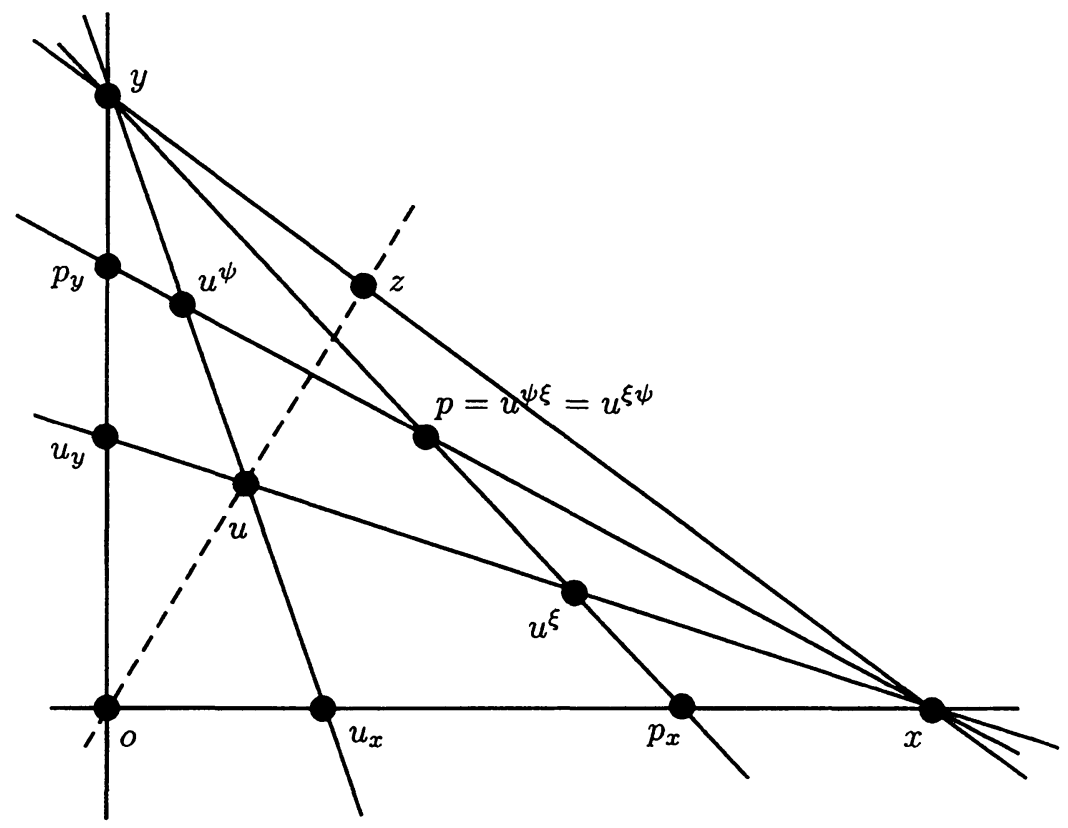

Figure 1. The action of $G$

Now put $z=o u \cap x y$ and let $Z$ be the stabilizer of $o u$. As $G$ fixes $o$ and is regular on ordinary points by Lemma $2.1, Z$ is a further subgroup of order $n-1$ of $G$. This implies severe restrictions on the structure of these groups which were first established by Hughes [14]; we state the following more general result proved by Sprague [26] in the context of translation nets.

Lemma 2.2. Let $G$ be a group of order $s^{2}$ with three pairwise disjoint subgroups $X, Y$ and $Z$ of order $s$, and assume that $X$ and $Y$ are normal. Then $X, Y$ and $Z$ are pairwise isomorphic. Moreover, $G$ is abelian if and only if $Z$ is also a normal subgroup.

Next, we note that our collineation group $G$ has the same orbit structure on $\Pi$ as a quasiregular group of type (g) in the Dembowski-Piper classification would have: the seven point orbits are

- the orbit of ordinary points on which $G$ acts regularly;

- the three fixed points $o, x$, and $y$;

- the $n-1$ points $z^{\prime} \neq x, y$ on $x y$, and similarly for the other two sides of the triangle oxy.

The line orbits are given dually. This poses the natural question under which conditions $G$ actually is quasiregular. We get the following result part of which is already contained in Hughes' work, see [14, Theorem 10], who would call $G$ a partially tran- 
sitive collineation group of type (3) for which two of the three distinguished subgroups defined in this case are normal.

Proposition 2.3. With the preceding notation, the following conditions are all equivalent:

(i) $G$ is a collineation group of Lenz-Barlotti type I.4.

(ii) $Z$ consists of $(o, x y)$-homologies.

(iii) $Z$ is a normal subgroup of $G$.

(iv) $G$ is quasiregular.

(v) $X$ is abelian.

(vi) $G$ is abelian.

Proof. As $Z$ is the stabilizer of $z$ in $G$ and acts regularly on the ordinary points on the line $o z$, it is clear that $G$ is of type I.4 if and only if all collineations in $Z$ are $(o, x y)$ homologies. This means that each element fixing $z$ has to fix every point on the line $x y$; as $G$ is transitive on the points $z^{\prime} \neq x, y$ on $x y$ and as the stabilizer of $z^{\gamma}$ is $\gamma^{-1} Z \gamma$, this happens if and only if $Z$ is a normal subgroup of $G$. This in turn is equivalent to saying that $G$ is quasiregular; for it is clear that $G$ induces a regular action on all other orbits, as $X$ and $Y$ consist of homologies. Finally, $X$ is abelian if and only if $G$ is; by Lemma 2.2 , this holds if and only if $Z$ is normal.

Thus a quasiregular group of type at least I.3 actually has type I.4, and so $\Pi$ is of Lenz-Barlotti class at least I.4 in this case. Note, however, that it is not clear at this point if the converse holds, i.e., if every group of type I.4 is quasiregular. It would a priori be conceivable that a third (then necessarily non-abelian) transitive group $U$ of $(o, x y)$-homologies exists, but that $U$ is not contained in the group $G=X \times Y$ generated by the other two homology groups we started with. This is actually not possible, as we will see soon.

Let us first discuss the known examples of planes admitting a group of type at least I.3. No examples of finite projective planes in Lenz-Barlotti class I.3 or I.4 are known, and it is widely conjectured that such planes do not exist; we shall discuss this problem later. In fact, all known finite planes with a group of type at least I.3 are defined over a nearfield $K$. Losely speaking, a proper nearfield may be thought of as a non-commutative field with only one distributive law; see [5] for the precise definition. We remark that the finite nearfields were completely classified by Zassenhaus [30], see also Dembowski $[5, \S 5.2]$. The given homology groups $X$ and $Y$ are isomorphic to the multiplicative group $K^{*}$. There arise two possibilities:

- If $K$ is proper, that is, not a field, $K^{*}$ is non-commutative. Then $\Pi$ has LenzBarlotti class IVa.2, unless $K$ is the exceptional nearfield of order 9 (in which case $\Pi$ has class IVa.3). Here the group $G=X \times Y$ is of type I.3 and is therefore not quasiregular. 
- If $K$ is a field, so that $K^{*}$ is commutative, then $\Pi$ is desarguesian and thus in LenzBarlotti class VII.2. Here the group $G=X \times Y$ is of type I.4 and quasiregular.

The preceding examples are already given in Hughes' paper [14], in terms of his partially transitive planes, though he could of course not yet discuss the connection to the Lenz-Barlotti classification, as Barlotti's paper only appeared a year later. These examples reappear in various later papers, usually without any specific reference to Hughes. It seems that his paper has been largely ignored, even though it is quite often cited in a rather vague general way. We want to advertise his work here, as it anticipates many of the ideas in the later Dembowski-Piper classification. Let us note that Hughes [14] also considered planes with a partially transitive group of type (3)-that is, with the same orbit structure as a quasiregular group of type (g) - without the assumption that at least two of the distinguished subgroups involved are normal. We decided not to deal with this case, as no examples of such groups seem to be known and as this would make our discussion more technical.

We now need to appeal to a standard approach for the study of projective planes, namely the introduction of coordinates. For this, we refer the reader to the exposition given by Dembowski [5] which essentially follows Hall [9]; this method of coordinatizing is not the only one in common use, cf. Hughes and Piper [15] and Pickert [23]. The resulting algebraic structure is called a Hall planar ternary ring. Assuming that $\Pi$ is $(y, o x)-,(x, o y)$ - and $(o, x y)$-transitive, i.e., at least in Lenz-Barlotti class I.4, coordinatizing yields a linear planar ternary ring $(R, T)$ such that

- $\left(R^{*}, \cdot\right)$ is a group, where $R^{*}=R \backslash\{0\}$;

- both distributive laws hold in $(R,+, \cdot)$ :

$(a+b) c=a c+b c, c(a+b)=c a+c b$ for all $a, b, c \in R$.

Following Kantor [17], such a planar ternary ring is called a neofield; earlier, the term planar division neo-ring (PDNR) was used by Hughes [12], [13]. ${ }^{2}$ The motivation for changing Hughes' terminology is given by the fact that finite neofields have the following additional properties:

- $(R,+)$ is commutative;

- $(a+b)+(-b)=a$ for all $a, b \in R$

- $\left(R^{*}, \cdot\right)$ is commutative.

The first two of these properties are due to Hughes extending earlier work of Paige [20], while the third one is due to Kantor and Pankin [18]; it is a generalization of Wedderburn's theorem. Thus we see that a finite neofield satisfies all of the field axioms except for the associativity of addition, which has been replaced by the so-called inverse property $(a+b)+(-b)=a$.

Conversely, every finite neofield coordinatizes a projective plane which is either of type I.4 (when the neofield is proper, i.e., not a field) or desarguesian. Given a neo-

\footnotetext{
${ }^{2}$ The term neofield was already used by Paige [20], but in a more general sense. His neofields are, in general, not associated with projective planes.
} 
field $R$, it is convenient to introduce an affine plane $\Sigma=\Sigma(R)$ in the usual way. Thus the points of $\Sigma$ are the ordered pairs $(a, b)$ with $a, b \in R$, and the lines are the point sets

$$
[m, k]=\{(r, r m+k): r \in R\} \quad \text { and } \quad[a]=\{(a, b): b \in R\}
$$

Coordinatizing the projective plane $\Pi$ corresponding to $\Sigma$ appropriately, we essentially recover the neofield $R$ we started with.

We can now demonstrate the result already announced in the discussion after Proposition 2.3:

Theorem 2.4. Let $\Pi$ be a finite projective plane. Then the following assertions are equivalent:

(a) $\Pi$ is in Lenz-Barlotti class at least I.4.

(b) $\Pi$ admits an abelian collineation group of Lenz-Barlotti type I.4.

(c) $\Pi$ admits a quasiregular collineation group of type ( $\mathrm{g}$ ).

Proof. Assume first that $\Pi$ is in Lenz-Barlotti class at least I.4, say $\Pi$ is $(y, o x)$-, $(x, o y)$ - and $(o, x y)$-transitive for the triangle oxy. Then $G=X \times Y$ is a collineation group of Lenz-Barlotti type at least I.3, where $X$ is the group of all $(x, o y)$ homologies and $Y$ the group of all $(y, o x)$-homologies, by Lemma 2.1. Now coordinatize $\Pi$ using a neofield $R$, as above. From the description of the affine part $\Sigma$, it is easily seen that the mapping $(a, b) \mapsto(a c, b)$ is an $(x, o y)$-homology for each $c \in R$. Hence the group $X$ of all these homologies is isomorphic to $R^{*}$ and therefore abelian, by the theorem of Kantor and Pankin [18]. Hence $G$ is abelian, and Proposition 2.3 shows that $G$ has Lenz-Barlotti type I.4. Again by Proposition 2.3, this implies that $\Pi$ admits a quasiregular collineation group $G$ of type $(\mathrm{g})$. Finally, assume that $G$ is such a group. Then the regularity of the actions induced on the three point orbits corresponding to the sides of the special triangle $o x y$ implies that the stabilizer of any point in one of these orbits consists of homologies, and thus $\Pi$ is obviously in LenzBarlotti class at least I.4.

\section{Neo-difference sets}

In this section, we discuss the representation of a finite projective plane in LenzBarlotti class at least I.3 by a certain type of difference set relative to disjoint subgroups in the sense of Hiramine [10] which we will call a "neo-difference set", as the abelian case turns out to correspond to planes in Lenz-Barlotti class at least I.4 and thus to neofields. We note that this type of difference set was first considered by Hughes [12], [13], [14]; in his terminology, it is a "partial difference set" for a partially transitive plane of type (3).

As usual in the study of any type of difference set, it is convenient to use the integral group ring $\mathbb{Z} G$. Let us recall the necessary notation. For $A=\sum a_{g} g \in \mathbb{Z} G$ 
and $t \in \mathbb{Z}$ we write $A^{(t)}=\sum a_{g} g^{t}$ and $[A]_{g}=a_{g}$ (the coefficient of $g$ in $A$ ). For $r \in \mathbb{Z}$ we write $r$ for the group ring element $r \cdot 1$, and for $S \subseteq G$ we write $S$ instead of $\sum_{g \in S} g$, by a convenient abuse of notation. Also, the following simple observation showing how to compute intersection sizes using $\mathbb{Z} G$ will be useful.

Lemma 3.1. Let $G$ be a finite abelian group and consider two subsets $A$ and $B$ of $G$. Then $|A \cap B g|=\left[A B^{(-1)}\right]_{g}$.

Using group ring notation, a neo-difference set of order $n$ may be defined to be a subset $D$ of a group $G$ of order $(n-1)^{2}$ with three pairwise disjoint subgroups $X, Y$, and $Z$ of order $n-1$ which satisfies the equation

$$
D D^{(-1)}=n+G-X-Y-Z
$$

in $\mathbb{Z} G$; thus every element $\gamma$ not in the union $N$ of the three forbidden subgroups $X, Y$, and $Z$ has a unique "difference representation" $\gamma=\delta \varepsilon^{-1}$ with $\delta, \varepsilon \in D$. In what follows, we shall only consider normal neo-difference sets; that is, we assume that at least two of the given subgroups, say $X$ and $Y$, are normal, so that Lemma 2.2 applies. We begin by constructing a normal neo-difference set from any finite projective plane in Lenz-Barlotti class at least I.3; for the convenience of the reader, we shall sketch the standard argument needed.

Proposition 3.2. Let $\Pi$ be a finite projective plane of order $n$ which is both (y,ox)-and $(x, o y)$-transitive, where $o, x$, and $y$ form a triangle, and define $G, X, Y$, and $Z$ as in Section 2. Then there exists a normal neo-difference set of order $n$ in $G$ with respect to the forbidden subgroups $X, Y$, and $Z$.

Proof. By Lemma 2.1, we may identify the image of the base point $u$ under the collineation $(\xi, \psi) \in G=X \times Y$ with the group element $(\xi, \psi)$. Now choose an ordinary line $D$ as base line. By the previous identification, we may consider $D$ as an $(n-2)$ subset of $G$; then the ordinary lines take the form $D \gamma$ with $\gamma \in G$. Now one easily checks that the number of lines of this form which join two given ordinary points $\left(\xi_{1}, \psi_{1}\right)$ and $\left(\xi_{2}, \psi_{2}\right)$ is the number of difference representations

$$
\left(\xi_{1}, \psi_{1}\right)\left(\xi_{2}, \psi_{2}\right)^{-1}=\left(\delta_{1}, \delta_{2}\right)\left(\varepsilon_{1}, \varepsilon_{2}\right)^{-1}
$$

with $\left(\delta_{1}, \delta_{2}\right),\left(\varepsilon_{1}, \varepsilon_{2}\right) \in D$. As $\Pi$ is a projective plane, this number is always 1 , unless $\left(\xi_{1}, \psi_{1}\right)$ and $\left(\xi_{2}, \psi_{2}\right)$ are on a line passing through one of the points $o, x$, and $y$, in which case it is 0 . These lines are precisely the right cosets of the three forbidden subgroups. Hence two points joined by a line passing through one of the points $o, x$, and $y$ have a difference in one the three subgroups $X, Y$, and $Z$, and $D$ is indeed a normal neo-difference set.

Our next aim is to establish the converse of Proposition 3.2. Thus let $D$ be a normal neo-difference set of order $n$, as defined above. We now make some simplifying 
assumptions. First of all, as $Y \cong X$, it may be replaced by $X$. Then $G=X \times X$, the three forbidden subgroups take the form

$$
U_{1}=X \times\{1\}, \quad U_{2}=\{1\} \times X, \quad U_{3}=\{(\xi, \xi): \xi \in X\}
$$

and equation (3.1) becomes

$$
D D^{(-1)}=n+G-U_{1}-U_{2}-U_{3}
$$

At this point, we note a simple restriction basically due to Paige [20].

Lemma 3.3. The group $X$ contains at most one involution.

Proof. Let $\gamma$ be an involution of $G$, and assume $\gamma \notin N=U_{1} \cup U_{2} \cup U_{3}$. Then there is a representation $\gamma=\delta \varepsilon^{-1}$ with $\delta, \varepsilon \in D$. But this implies the second representation $\gamma=\gamma^{-1}=\varepsilon \delta^{-1}$, a contradiction. Hence all involutions of $G$ are contained in $N$. Now let $\kappa$ and $\lambda$ be involutions of $X$. Then $(\kappa, \lambda)$ is an involution of $G$ and thus lies in $N$, forcing $\kappa=\lambda$.

We continue with our simplifying assumptions. Note that, for $i=1,2,3$, there is exactly one coset of $U_{i}$ which misses $D$, whereas every other coset intersects $D$ uniquely, as no element in $N$ has a difference representation from $D$. By replacing $D$ with a suitable translate, we may assume that both $U_{1}$ and $U_{2}$ miss $D$. Let us write the unique coset of $U_{3}$ missing $D$ in the form $U_{3}(1, \theta)$ with $\theta \in X$; we will later determine the value of $\theta$ if $G$ is abelian. With these assumptions, we may write

$$
D=\sum_{\xi \in X \backslash\{1\}}(\xi, g(\xi)),
$$

where $g: X \backslash\{1\} \rightarrow X \backslash\{1\}$ is a bijection. Note that the element $(\xi, g(\xi))$ is in the coset $U_{3}\left(1, \xi^{-1} g(\xi)\right)$, and therefore

$$
\Delta:=\left\{\xi^{-1} g(\xi): \xi \in X\right\}=X \backslash\{\theta\}
$$

We can now give an explicit description of the desired projective plane $\Pi=\Pi(D)$ in terms of $D$, see Figure 2. For this, we choose an element $0 \notin X$ and embed $X$ into the semigroup $\bar{X}=X \cup\{0\}$, where $0 \xi=\xi 0=0$ for all $\xi \in X$, as well as a further symbol $\infty \notin \bar{X}$. The points of $\Pi$ are

- the $n^{2}$ elements $(\xi, \psi) \in \bar{G}=\bar{X} \times \bar{X}$;

- $n$ points $(\xi)$, where $\xi \in \bar{X}$, and a point $(\infty)$;

and the lines of $\Pi$ are

- $(n-1)^{2}$ lines $[\xi, \psi]=D(\xi, \psi) \cup\left\{(\xi, 0),(0, \psi),\left(\theta \psi \xi^{-1}\right)\right\}$ with $\xi, \psi \in X$; 


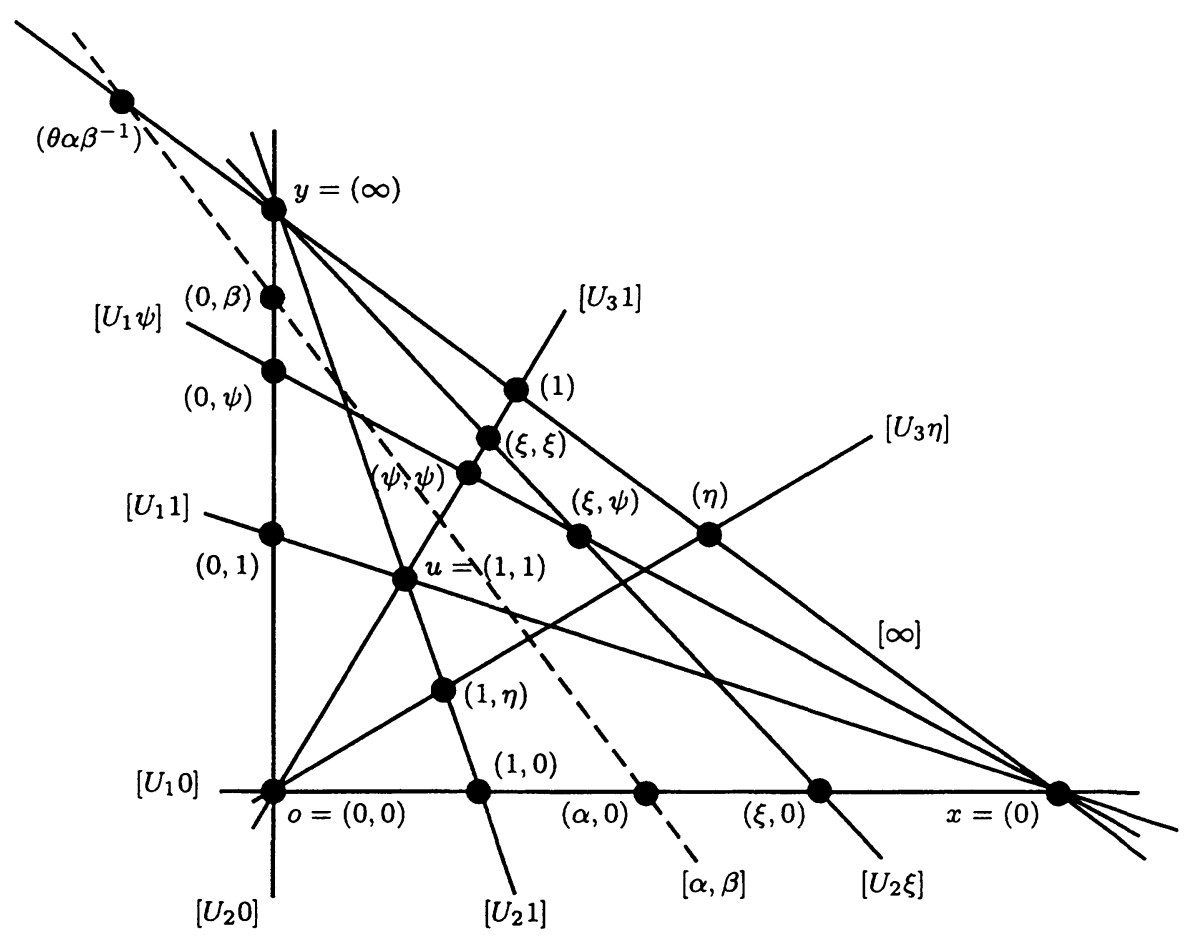

Figure 2. The plane $\Pi(D)$

- $n$ lines $\left[U_{1} \psi\right]=\{(\xi, \psi): \xi \in \bar{X}\} \cup\{(0)\}$, where $\psi \in \bar{X}$;

- $n$ lines $\left[U_{2} \xi\right]=\{(\xi, \psi): \psi \in \bar{X}\} \cup\{(\infty)\}$, where $\xi \in \bar{X}$;

- $n-1$ lines $\left[U_{3} \psi\right]=\{(\xi, \xi \psi): \xi \in \bar{X}\} \cup\{(\psi)\}$, where $\psi \in X$;

- a line $[\infty]=\{(\xi): \xi \in \bar{X}\} \cup(\infty)$.

The preceding construction is inspired on one hand by the work of Hughes, cf. [14, pp. 660-662], with some simplifications made possible by the more special situation we consider here, and on the other hand, by the neofield representation of planes of type I.4 discussed in the previous section; it is also similar (but more involved) to the presentation of planes with a quasiregular group of type (f) as given by de Resmini and the present authors [4].

Proposition 3.4. The incidence structure $\Pi=\Pi(D)$ defined above is a projective plane of order $n$, and $G$ acts on $\Pi$ as a collineation group of Lenz-Barlotti type at least I.3.

Proof. Clearly $G$ acts on $\Pi$ by right translation. Let us put $o=(0,0), x=(0)$ and $y=(\infty)$ and call points $(\xi, \psi) \in X \times X$ ordinary. Then the orbit structure of $G$ on points is as described in Section 2. As $\Pi$ has $n^{2}+n+1$ points and $n^{2}+n+1$ lines 
and as each line has $n+1$ points, it suffices to check that any two points $p, q$ of $\Pi$ are joined by at least one line. In several cases, this is trivial to see, namely if one of the points is $o=(0,0), x=(0)$ or $y=(\infty)$ or if both points are of one of the forms $(\xi, 0),(0, \psi)$ and $(\xi)$. Thus we may assume that $p$ is an ordinary point. Because of the transitivity of $G$ on such points, we may also assume $p=(1,1)$. Then $p$ is joined to every point of the form $(\xi, 1)$ by the line $\left[U_{1} 1\right]$, to every point of the form $(1, \psi)$ by the line $\left[U_{2} 1\right]$, and to every point of the form $(\xi, \xi)$ by the line $\left[U_{3} 1\right]$. If $q$ is an ordinary point which is not on one of these three lines, say $q=(\xi, \psi)$, then the "difference" $(\xi, \psi)(1,1)^{-1}$ determined by $q$ and $p$ is in $G \backslash N$, and the argument given in the proof of Proposition 3.2 shows that $p$ and $q$ are joined by a unique ordinary line, i.e., a line of the type $[\alpha, \beta]$, as $D$ is a neo-difference set. This leaves us with the case where $q$ is on a side of the triangle $o x y$. We now make use of the form of $D$ given in equation (3.3) and note that the line $\left[\xi^{-1}, g(\xi)^{-1}\right]$ contains both $p=(1,1)$ and the points $\left(\xi^{-1}, 0\right),\left(0, g(\xi)^{-1}\right)$ and $\left(\theta g(\xi)^{-1} \xi\right)$. As $g: X \backslash\{1\} \rightarrow X \backslash\{1\}$ is a bijection, we see that $p$ is joined to every point on $o x$ except for $(1,0)$ and to every point on oy except for $(0,1)$ by one of these lines. Moreover, $p$ is also joined to every point on $x y$ except for (1), as the set of elements $\xi^{-1} g(\xi) \theta^{-1}$ is $X \backslash\{1\}$, by equation (3.4). But the three exceptional points are taken care of by the lines $\left[U_{i} 1\right]$ through $p$. Finally, it is trivial to check that the elements in $U_{1}$ and $U_{2}$ act as homologies on $\Pi$ so that $\Pi$ is indeed $(y, o x)$ - and $(x, o y)$-transitive.

The incidence structure $\mathscr{D}$ formed by the ordinary points and lines is what we might call a triangular semiplane admitting $G=X \times X$ as a Singer group. As we have seen, $\Pi$ can be reconstructed uniquely from $\mathscr{D}$. More generally, it is known that a geometry which looks like a projective plane with a triangle removed actually is such a structure provided that the order is at least 25 , see Ralston [24].

Example 3.5. Let $K$ be a finite nearfield of order $n$. Then the set

$$
D=\left\{(\xi, \psi) \in K^{*} \times K^{*}: \xi+\psi=1\right\}
$$

is a normal neo-difference set of order $n$ in $G=K^{*} \times K^{*}$, as it is easily checked directly, using the axioms of a nearfield. This example is due to Hughes [14, pp. 656-657] and was re-discovered by Hiramine [10, Example 4.2.(iv)]. Note that $D$ is abelian if and only if $K$ is actually a finite field. Accordingly, the projective plane associated with $D$ as in Proposition 3.4 is either a nearfield plane or desarguesian. In this way, we obtain the known examples of planes admitting a group of LenzBarlotti type at least I.3 discussed at the end of Section 2.

Propositions 3.2 and 3.4 together establish the first main result of this section:

Theorem 3.6. A finite projective plane $\Pi$ admits a collineation group $G$ of Lenz-Barlotti type at least I.3 if and only if it can be represented by a normal neo-difference set.

Combining this with Theorem 2.4, we also have the following second main result: 
Theorem 3.7. Let $\Pi$ be a finite projective plane. Then the following assertions are equivalent:

(a) $\Pi$ is in Lenz-Barlotti class at least I.4.

(b) $\Pi$ admits an abelian collineation group of Lenz-Barlotti type I.4.

(c) $\Pi$ admits a quasiregular collineation group of type (g).

(d) $\Pi$ can be represented by an abelian neo-difference set.

We next note two interesting restrictions on the structure of abelian neo-difference sets for which a more direct proof (avoiding the use of the associated neofield) would be desirable; unfortunately, this has eluded us.

Proposition 3.8. Let $D$ be an abelian neo-difference set of order $n$, as in equation (3.2). Then $D$ may be assumed to be symmetric with the inverse property, in the sense that $(\xi, \psi) \in D$ implies both $(\psi, \xi) \in D$ and $\left(\xi^{-1}, \varepsilon \xi^{-1} \psi\right) \in D$, where $\varepsilon$ is the unique involution in $X$ if $n$ is odd and $\varepsilon=1$ otherwise, cf. Lemma 3.3.

Proof. By Proposition 3.4, $D$ gives rise to a projective plane $\Pi$ in Lenz-Barlotti class at least I.4 on which the underlying group $G$ acts by right translation. If we coordinatize $\Pi$ as in the proof of Proposition 3.2 and use $[1,1]$ as base line, we recover $D$. On the other hand, we may also coordinatize $\Pi$ using a neofield $R$, as discussed in Section 3. Then the homology groups $X$ and $Y$ can be identified with the subgroups $R^{*} \times\{1\}$ and $\{1\} \times R^{*}$ of $R^{*} \times R^{*} \cong G \cong X \times X$. Hence the coordinates of ordinary points agree in both the neofield and the neo-difference set setting, if we identify $X$ with $R^{*}$. Now let us consider the affine line

$$
L=[-1,1]=\{(r,-r+1): r \in R\}=\{(\xi, \psi) \in R \times R: \xi+\psi=1\}
$$

If we choose the line $L$ as the base line in determining $D$ (so we replace the original $D$ by a suitable translate, if necessary), the commutativity of the addition in $R$ immediately implies the symmetry of $D$.

Regarding the inverse property, we first note that $1+(-1)=0$ in $R$ implies $\alpha+(-1) \alpha=0$ and thus $-\alpha=(-1) \alpha$ for all $\alpha \in R$, as expected; in particular, $(-1)^{2}=$ 1. This shows-still identifying $X$ with $R^{*}$-that -1 is just the element $\varepsilon$ defined in the assertion. Multiplying the equation $\xi+\psi=1$ by $\xi^{-1}$, we get $1+\xi^{-1} \psi=\xi^{-1}$ which is equivalent to $\xi^{-1}+\varepsilon \xi^{-1} \psi=1$.

At a later point, we shall require a characterization of the elements of order 3. For this, it is more convenient to rephrase the inverse property in terms of the function $g$ introduced in (3.3) as follows:

$$
g\left(\xi^{-1}\right)=\varepsilon \xi^{-1} g(\xi) \text { for all } \xi \in X
$$


Corollary 3.9. Under the assumptions of Proposition 3.8, an element $\alpha \in X$ has order 3 if and only if the following condition holds:

$$
g(\varepsilon \alpha)=\varepsilon \alpha^{2} \quad \text { and } \quad g\left(\varepsilon \alpha^{2}\right)=\varepsilon \alpha
$$

Proof. First note that the two equations in (3.6) are equivalent by the symmetry of $D$. Assuming that these equations hold, we compute

$$
\alpha^{2} g\left(\varepsilon \alpha^{2}\right)=\alpha^{2} \varepsilon \alpha=\alpha \varepsilon \alpha^{2}=\alpha g(\varepsilon \alpha)=\alpha^{2} g\left(\varepsilon \alpha^{-1}\right)
$$

where the last equality follows by applying equation (3.5) to the element $\xi=\varepsilon \alpha$. As $g$ is a bijection, we immediately conclude $\alpha^{2}=\alpha^{-1}$ so that $\alpha$ indeed has order 3. Conversely, let $\alpha$ be any element of order 3. Applying equation (3.5) to the element $\xi=\varepsilon \alpha$, we get the identity $g\left(\varepsilon \alpha^{2}\right)=\alpha^{2} g(\varepsilon \alpha)$ which allows us to obtain a "repeated difference" from $D$ :

$$
\begin{aligned}
(\varepsilon \alpha, g(\varepsilon \alpha)) \cdot\left(g\left(\varepsilon \alpha^{2}\right), \varepsilon \alpha^{2}\right)^{-1} & =\left(\varepsilon \alpha g\left(\varepsilon \alpha^{2}\right)^{-1}, g(\varepsilon \alpha) \varepsilon \alpha\right) \\
& =\left(\varepsilon \alpha^{2} g(\varepsilon \alpha)^{-1}, g\left(\varepsilon \alpha^{2}\right) \varepsilon \alpha^{2}\right) \\
& =\left(\varepsilon \alpha^{2}, g\left(\varepsilon \alpha^{2}\right)\right) \cdot(g(\varepsilon \alpha), \varepsilon \alpha) .
\end{aligned}
$$

As $\varepsilon \alpha \neq \varepsilon \alpha^{2}$, we conclude $\left(\varepsilon \alpha g\left(\varepsilon \alpha^{2}\right)^{-1}, g(\varepsilon \alpha) \varepsilon \alpha\right)=(1,1)$, as desired.

\section{Ovals associated with abelian neo-difference sets}

In this section, we show that any finite plane associated with an abelian neo-difference set admits a system of ovals forming an interesting configuration. This is similar to our work on ovals in planes admitting a quasiregular group of type (f) in [4], where the possibility of such an approach was mentioned but-in view of the technical effort needed-not considered interesting enough to be carried through. As we shall see, some interesting consequences do emerge after all; also, we have all the machinery needed ready by now.

Proposition 4.1. Let $\Pi$ be a projective plane of order $n$ represented by a neo-difference set $D$ in an abelian group $G$, as in Section 3, and let $D$ have the form (3.3). Then the $(n-2)$-sets $A_{\gamma}=D^{(-1)} \gamma$ with $\gamma=(\alpha, \beta) \in G$ are arcs in $\Pi$, and the line $\left[\xi^{-2} \alpha, g(\xi)^{-2} \beta\right]$ is the tangent to $A_{\gamma}$ with $(\xi, g(\xi))^{-1} \gamma$ as the tangency point. Moreover, the $(n-2)$-arc $A_{\gamma}$ may be extended to an oval of $\Pi$, namely $O_{\gamma}=A_{\gamma} \cup\{(0,0),(0),(\infty)\}$. Finally, if $n$ is even, the nucleus of $O_{\gamma}$ is the ordinary point $\gamma$.

Proof. The proof uses standard arguments as in [4], and hence we will just give a sketch. Clearly, no coset of one of the three forbidden subgroups $U_{i}$ intersects a translate of $D$ in more than one point. Also, each set $D \kappa$ intersects $A_{\gamma}=D^{(-1)} \gamma$ at 
most twice, and one gets a unique point of intersection if and only if $\kappa=\delta^{-2} \gamma$. As a line of $\Pi$ through one of the points $o=(0,0), x=(0)$, and $y=(\infty)$ intersects $A_{\gamma}$ at most once, $O_{\gamma}$ is an oval. Finally, if $n$ is even, all the tangents of $O_{\gamma}$ have to be concurrent. For $\gamma=(1,1)$, the lines $\left[U_{1} 1\right]$ and $\left[U_{2} 1\right]$ do not meet $D^{(-1)}$ and therefore are tangents of $O_{(1,1)}$. But these two lines meet in the ordinary point $(1,1)$ which has to be the nucleus of $O_{(1,1)}$. Now the transitivity of $G$ shows that $\gamma$ is the nucleus of $O_{\gamma}$ in general.

We note two interesting consequences of Proposition 4.1. The first of these has been proved by Kantor [17] in a different way, and the second one is the determination of the exceptional group element $\theta$ defined in Section 3.

Corollary 4.2. Assume that $n \neq 2$ is even. Then $n$ is a multiple of 4 .

Proof. Note that $D$ is disjoint from any translate of the form $D \gamma$ with $1 \neq \gamma \in N$. For such a $\gamma$, the hyperovals completing $O_{(1,1)}$ and $O_{\gamma}$ intersect precisely in the three special points $(0,0),(0)$ and $(\infty)$. But in a plane of order $n \equiv 2(\bmod 4)$ any two hyperovals have to intersect in an even number of points; see, for instance, [16, Lemma 3.3].

Proposition 4.3. Let $D$ be a neo-difference set of order $n$ in an abelian group $G=$ $X \times X$, as in (3.3), and assume that $D$ misses the coset $U_{3}(1, \theta)$ so that $\theta$ satisfies equation (3.4). Then $\theta=1$ provided that $n$ is even; otherwise, $\theta$ is the unique involution in $X$.

Proof. We consider the oval $O=O_{(1,1)}$. First let $n$ be even. Then the nucleus of $O$ is the point $(1,1)$, by Proposition 4.1. Obviously, the line $\left[U_{3} \theta\right]$ is the unique tangent of $O$ in the point $(0,0)$; therefore, the coset $U_{3} \theta$ has to contain $(1,1)$, and hence $\theta=1$. From now on, let $n$ be odd. Then $\theta \neq 1$, as the point $(1,1)$ lies on the two tangents $\left[U_{1} 1\right]$ and $\left[U_{2} 1\right]$ and cannot be on a further tangent. Therefore $D$ meets $U_{3}$, and hence $O$ contains a (unique) point of the form $\left(\xi^{-1}, \xi^{-1}\right)$, that is, $g$ fixes a unique element $\xi_{0} \in X$. By Proposition 4.1, the line $L_{\xi}=\left[\xi^{-2}, g(\xi)^{-2}\right]$ is the unique tangent of $O$ in the point $(\xi, g(\xi))$, where $\xi$ runs over $X \backslash\{1\}$. By definition, $L_{\xi}$ intersects $[\infty]=x y$ in the point $\left(\theta g(\xi)^{-2} \xi^{2}\right)$. In particular, the tangent $L_{\xi_{0}}$ intersects $\infty$ in $(\theta)$. But the tangent $\left[U_{3} \theta\right]$ also contains $(\theta)$, and hence $(\theta)$ cannot be on any further tangent. Now assume that $\theta$ is not the unique involution $\tau \in X$, so that $\tau=\xi^{-1} g(\xi)$ for some $\xi \in X$. Then the corresponding tangent $L_{\xi}$ intersects $[\infty]$ in $\left(\theta g(\xi)^{-2} \xi^{2}\right)=\left(\theta \tau^{-2}\right)=(\theta)$ and we have found a third tangent through $(\theta)$, a contradiction.

Let us also mention the following constructive result which is immediate from Proposition 4.1. In the special case $\Pi=\mathrm{PG}(2, n), n$ odd, it reduces to a known statement on conic sections, by the theorem of Segre [25].

Proposition 4.4. Let $\Pi$ be a projective plane of order $n$ represented by a neo-difference set $D$ in an abelian group $G$, as in Section 3. Then $\Pi$ contains a family $\mathcal{O}$ of $(n-1)^{2}$ 
ovals all of which contain the special triangle oxy and pairwise have at most one further point of intersection.

Finally, the proof of Proposition 4.3 suggests a further interesting geometric application. Recall that a projective triangle of side $k$ in a plane of order $n$ is a set $B$ of $3(k-1)$ points with the following properties:

(a) $B$ contains a distinguished triangle $o x y$.

(b) On each side of $o x y$, there are exactly $k$ points of $B$.

(c) If the points $q \in o x$ and $r \in o y$ belong to $B$, then $q r \cap x y$ also belongs to $B$.

We now show that planes with a group of type at least I.4 contain projective triangles forming small blocking sets; see Hirschfeld [11, Chapter 13] for background.

Proposition 4.5. Let $\Pi$ be a projective plane of odd order $n$ represented by a neodifference set $D$ in an abelian group $G$, as in Section 3. Let $O$ denote the oval $D^{(-1)} \cup\{o, x, y\}$, where $o=(0,0), x=(0)$, and $y=(\infty)$, and define $B$ as the set of all points which arise as the intersection of some side of oxy with some tangent of $O$. Then $B$ is a projective triangle of side $\frac{1}{2}(n+3)$ which is a minimal blocking set for $\Pi$.

Proof. We use Proposition 4.3 and the facts observed in its proof. The line $L_{\xi}$ meets the $x$-axis $o x$ in $\left(\xi^{-2}, 0\right)$, the $y$-axis oy in $\left(0, g(\xi)^{-2}\right)$, and the line at infinity $x y$ in $\left(\theta g(\xi)^{-2} \xi^{2}\right)$. Hence

$$
B=\{o, x, y\} \cup\left\{(\xi, 0): \xi \in X^{\square}\right\} \cup\left\{(0, \psi): \psi \in X^{\square}\right\} \cup\left\{(\theta \eta): \eta \in X^{\square}\right\},
$$

where we write $X^{\square}$ for the set of squares in $X$. As $X$ contains a unique involution by Lemma 3.3, $X^{\square}$ has index 2 in $X$ which shows that condition (b) above is satisfied. Consider a point $q=(\xi, 0) \in o x$ and a point $r=(0, \psi) \in o y$. Then $q r$ is the line $[\xi, \psi]$ and thus $z=q r \cap x y=\left(\theta \psi \xi^{-1}\right)$. Hence $q, r \in B$ implies $z \in B$, and $B$ is indeed a projective triangle. On the other hand, if the line $L=[\xi, \psi]$ intersects neither $o x$ nor $o y$ in a point of $B$, then both $\xi$ and $\psi$ must be non-squares. As $X^{\square}$ has index 2 in $X$, we see that $\theta \psi \xi^{-1}$ is then also a non-square. Thus $L$ intersects $x y$ in a point of $B$, so that $B$ is indeed a blocking set which is obviously minimal.

In the special case of desarguesian planes of odd order, the existence of projective triangles is well-known. But the proof and the geometric description provided above are new even in this case. More precisely, we obtain the following synthetic construction for projective triangles:

Corollary 4.6. Let $C$ be a conic in $\Pi=\mathrm{PG}(2, q)$, where $q$ is odd. Choose a triangle oxy contained in $C$, and let $B$ be the set of all points arising as the intersection of some side of oxy with some tangent of $C$. Then $B$ is a projective triangle of side $\frac{1}{2}(q+3)$ which is a minimal blocking set for $\Pi$. 


\section{Nonexistence results}

In this section, we establish some nonexistence results for abelian neo-difference sets, both old and new; our proofs are different from previous ones, as they do not make use of the associated neofield. These methods do not apply to the non-abelian case; for this reason, we refer the reader to the literature as far as nonexistence results for planes with a group of Lenz-Barlotti type I.3 are concerned, see e.g. Kantor [17] and Yaqub [29]. We begin with a structural restriction due to Paige [20], Hughes [13] and Kantor [17].

Theorem 5.1. Let $D$ be an abelian neo-difference set in $G=X \times X$. Then $X$ has cyclic Sylow 2- and 3-subgroups.

Proof. The Sylow 2-subgroup of $X$ is cyclic by Lemma 3.3. Now let $\alpha, \beta \in X$ be elements of order 3. By Corollary 3.9, we have

$$
g(\varepsilon \alpha)=\varepsilon \alpha^{2}, \quad g\left(\varepsilon \alpha^{2}\right)=\varepsilon \alpha, \quad g(\varepsilon \beta)=\varepsilon \beta^{2}, \quad g\left(\varepsilon \beta^{2}\right)=\varepsilon \beta .
$$

Using this, we obtain a "repeated difference" from $D$ :

$$
\left(\varepsilon \alpha, \varepsilon \alpha^{2}\right) \cdot\left(\varepsilon \beta, \varepsilon \beta^{2}\right)^{-1}=\left(\alpha \beta^{2}, \beta \alpha^{2}\right)=\left(\varepsilon \beta^{2}, \varepsilon \beta\right) \cdot\left(\varepsilon \alpha^{2}, \varepsilon \alpha\right)^{-1}
$$

and therefore $\beta \in\left\{\alpha, \alpha^{2}\right\}$. Thus $X$ indeed contains at most one subgroup of order 3 .

Next we consider multipliers. As usual in the theory of difference sets, we shall define a multiplier of an abelian neo-difference set $D$ of order $n$ as an automorphism $\alpha$ of the underlying group $G$ inducing a collineation of the associated projective plane $\Pi$. A multiplier of the special form $\alpha: x \mapsto t x$ for some integer $t$ with $\left(t,(n-1)^{2}\right)=1$ is called a numerical multiplier; by abuse of language, $t$ itself is also said to be a multiplier. It is clear that $\alpha \in$ Aut $G$ is a multiplier if and only if $\alpha(D)=D \gamma$ for some $\gamma \in G$.

All of the following results parallel corresponding statements for planar and affine difference sets, cf. [7], and therefore we have named some of them correspondingly. For instance, the proof of [2, Lemma VI.2.5] carries over to establish the following simple fact.

Lemma 5.2. Let $D$ be an abelian neo-difference set in $G$. Then there is an element $\gamma \in G$ such that $D \gamma$ is fixed by every multiplier.

We now prove a multiplier theorem first established by Hughes [13] using neofields. Hughes's original proof needed several pages and was rather technical and not very illuminating. In analogy to the cases of planar and affine difference sets discussed in [7], we provide a new proof which is much shorter and also more transparent. 
Theorem 5.3 (Multiplier theorem). If $D$ is an abelian neo-difference set of order $n$, then every prime divisor $p$ of $n$ is a multiplier of $D$. More precisely, we may assume $D=D^{(p)}$ in $\mathbb{Z} G$ for every prime $p$ dividing $n$.

Proof. We use the integral group ring $\mathbb{Z} G$ and assume w.l.o.g. that $D$ satisfies equation (3.2) and has the form (3.3). We first note the following auxiliary equations:

$$
D G=(n-2) G, \quad D U_{1}=G-U_{1}, \quad D U_{2}=G-U_{2} \quad \text { and } \quad D U_{3}=G-U_{3} \theta,
$$

where $\theta$ is as in equation (3.4) and has been determined explicitly in Proposition 4.3. We now claim

$$
\left|D^{(p)} \cap D g\right| \geqslant 1 \quad \text { for all } g \in G \backslash N,
$$

where $N=U_{1} \cup U_{2} \cup U_{3}$; trivially, this follows from the congruence

$$
\left|D^{(p)} \cap D g\right| \equiv 1 \quad \bmod p \quad \text { for all } g \in G \backslash N
$$

which we will prove using Lemma 3.1. Thus we evaluate the group ring element $D^{(p)} D^{(-1)}$ modulo $p$. Using the hypothesis $p \mid n$, equation (3.2), the auxiliary equations above and the well-known fact

$$
D^{p} \equiv D^{(p)} \quad \bmod p \quad \text { for } D \in \mathbb{Z} G
$$

which follows from the multinomial theorem, see [2, Lemma VI.3.7], we compute in $\mathbb{Z}_{p} G$ as follows:

$$
\begin{aligned}
D^{(p)} D^{(-1)} & =D^{p} D^{(-1)}=D^{p-1}\left(D D^{(-1)}\right) \\
& =D^{p-1}\left(G-U_{1}-U_{2}-U_{3}\right) \\
& =D^{p-2}\left(-2 G-\left(U_{1}-G\right)-\left(U_{2}-G\right)-\left(U_{3} \theta-G\right)\right) \\
& =D^{p-2}\left(G-U_{1}-U_{2}-U_{3} \theta\right) \\
& =\cdots \\
& =G-U_{1}-U_{2}-U_{3} \theta^{p-1}=G-U_{1}-U_{2}-U_{3} .
\end{aligned}
$$

This implies the desired congruence (5.2) and therefore (5.1). Up to now, we have established that all lines $[\xi, \psi]$ with $\gamma=(\xi, \psi) \notin N$ meet the set $D^{(p)}$. Now we note that all lines $[\xi, 1]$ contain the point $(0,1)$, all lines $[1, \psi]$ contain the point $(1,0)$, and all lines $[\xi, \xi]$ contain the point $(\theta)$. Hence all ordinary lines meet the set $L=$ $D^{(p)} \cup\{(0,1),(1,0),(\theta)\}$. Moreover, each of the three lines $\left[U_{1} 1\right],\left[U_{2} 1\right]$ and $\left[U_{3} \theta\right]$ contains one of the points $(0,1),(1,0)$ and $(\theta)$; for all other cosets of one of the three forbidden subgroups, the corresponding line $\left[U_{i} \xi\right]$ intersects $D$ and hence also $D^{(p)}$. Thus $L$ has $n+1$ points and meets every line of $\Pi$. Therefore, $L$ is itself a line of $\Pi$, 
by a well-known result due to Lander, see [2, Lemma VI.4.2]. Obviously, this means $L=[1,1]$ and hence $D=D^{(p)}$.

As the next five results show, multipliers of even order are of particular importance. These results are essentially due to Kantor [17] who used the language of neofields; thus our proofs will be rather different. The key result is the following characterization of multipliers of order 2; the geometric argument we give was inspired by the proof of the analogous statement for planar abelian difference sets due to Blokhuis, Brouwer and Wilbrink [3].

Theorem 5.4. Let $D$ be an abelian neo-difference set of order $n$ in $G$. If $D$ admits a multiplier $t$ of order 2 , then $n$ is a perfect square, say $n=m^{2}$, and necessarily $t=m$.

Proof. Let $t$ be any multiplier of order 2 of $D$, and denote the induced collineation of the associated projective plane $\Pi$ described in $\S 3$ by $\pi$. Then $\pi$ is an involution whose set of fixed points contains the quadrangle $o x y u$, where $u=(1,1)$. Thus $\pi$ is a Baer involution, that is, the fixed elements of $\pi$ form a Baer subplane $\Pi_{0}$; see Hughes and Piper [15]. In particular, $n$ must be a square, say $n=m^{2}$. We now define subgroups $A$ and $B$ of $X$ as follows:

$$
A=\left\{\xi \in X: \xi^{t}=\xi^{-1}\right\} \quad \text { and } \quad B=\left\{\xi \in X: \xi^{t}=\xi\right\} .
$$

Then the mappings $\alpha$ and $\beta$ defined by $\xi^{\alpha}=\xi^{1-t}$ and $\xi^{\beta}=\xi^{1+t}$ are homomorphisms from $X$ to $A$ and $B$, respectively, and $\xi^{\alpha} \xi^{\beta}=\xi^{2}$ for each $\xi \in X$; thus $A B=X^{\square}$ is the set of squares in $X$ and therefore a subgroup of index at most 2, by Lemma 3.3. As the ordinary points of $\Pi_{0}$ are simply the pairs $(\xi, \psi)$ with $\xi, \psi \in B$, we see that $B$ is the unique subgroup of order $m-1$ of $X$. It now follows from $A B=X^{\square}$ that $A$ must be the unique subgroup of order $m+1$ of $X$. (If $m$ is even, $A \cap B=\varnothing$, and otherwise $A \cap B=\{1, \varepsilon\}$, where $\varepsilon$ is the unique involution in $X$.) Therefore any multiplier of order 2 leads to the same subgroups $A$ and $B$ and acts on them in the same way as $t$ does. In particular, this holds for the multiplier $m$ of order 2 whose existence is guaranteed by Theorem 5.3. So the collineations induced by $t$ and $m$ agree on all ordinary points $(\xi, \psi)$ with $\xi, \psi \in X^{\square}$, and hence $t^{-1}$ must be the identity, proving $t=m$.

Corollary 5.5. Let $D$ be an abelian neo-difference set in $G$. Then the multiplier group of $D$ has a cyclic Sylow 2-subgroup.

Corollary 5.6. Let $D$ be an abelian neo-difference set of square order $n$ in $G$, say $n=m^{2}$. Then there also exists an abelian neo-difference set of order $m$.

Proof. By Theorem 5.3, $D$ is fixed by the multiplier $m$ of order 2. Hence, using the notation of the proof of Theorem 5.4, $D$ belongs to the Baer subplane $\Pi_{0}$ formed by the fixed elements of the collineation $\pi$ induced by $m$. Thus $D \cap B$ is an $(m-1)$ subset of $B \times B$ which is easily seen to be a sub-neo-difference set of $D$. 
As a consequence of Theorem 5.4, we obtain some useful restrictions:

Theorem 5.7 (Mann test). Let $D$ be an abelian neo-difference set of order $n$ in $G=$ $X \times X$. Then either $n$ is a square or every multiplier of $D$ has odd order modulo $\exp G$. In particular, each of the following conditions implies that $n$ is a square:

(a) D has a multiplier which has even order modulo $q$, where $q$ divides $n-1$ and either $q=4$ or $q$ is an odd prime;

(b) $p$ is a quadratic non-residue modulo $q$, where $p$ and $q$ are prime divisors of $n$ and of $n-1$, respectively;

(c) $n \equiv 4$ or $6(\bmod 8)$;

(d) $t p^{f} \equiv-1(\bmod q)$ for some prime $p$ dividing $n$, a suitable non-negative integer $f$ and some multiplier $t$ of $D$, where $q$ divides $n-1$ and either $q=4$ or $q$ is an odd prime;

(e) $(t+1, n-1) \geqslant 3$ for some multiplier $t$ of $D$.

Proof. If $t$ has even order, a suitable power of $t$ has order 2, and thus the first assertion is an immediate consequence of Theorem 5.4. Any multiplier which has even order $\bmod q$ also has even order modulo the exponent $v^{*}$ of $G$; this establishes (a). Then (b) follows from the observation that every quadratic non-residue has even order modulo $q$. Now assume $n \equiv 4$ or $6(\bmod 8)$; then $n$ is even and $n-1 \equiv 3$ or $5(\bmod 8)$. Therefore 2 is a quadratic non-residue modulo $n-1$, and thus there exists a prime divisor $q$ of $n-1$ such that 2 is also a quadratic non-residue modulo $q$. We may now choose $p=2$ to see that (c) is just a special case of (b). As for (d), tp $p^{f}$ is a multiplier that clearly has even order $\bmod q$; this is clear if $q$ is an odd prime, and follows for $q=4$, as the Sylow 2-subgroup of $X$ is cyclic. Thus (d) is a special case of (a). Finally, (e) is contained in (d), as $(t+1, n-1)$ either is a multiple of 4 or has an odd prime divisor.

We mention two examples which show how the Mann test may be applied; further results in the same spirit can be found in Kantor's paper [17].

Example 5.8. Assume the existence of an abelian neo-difference set of order $n \equiv 9$ $(\bmod 12)$. Then $t=3$ is a multiplier for which $t+1$ divides $n-1$, and thus $n$ is a square, by criterion (e).

Corollary 5.9. Let $D$ be an abelian neo-difference set of even order $n$. Then $n=2$, $n=4$, or $n$ is a multiple of 8 .

Proof. Assume $n \neq 2$. Then $n$ is a multiple of 4 , by Corollary 4.2. Now assume $n \equiv 4(\bmod 8)$. Then $n$ is a perfect square by Theorem 5.7, say $n=m^{2}$. By Corollary 5.6, there also exists an abelian neo-difference set of order $m$. As $m$ is even and not a multiple of 4 , we conclude $m=2$, by another application of Corollary 4.2. Thus $n$ is divisible by 8 whenever $n \neq 2$ or 4 . 
We also mention a simple nonexistence result due to Pankin [21] which should be compared to criterion (d) of Theorem 5.7.

Proposition 5.10. No abelian neo-difference set of order $n>4$ has -1 as a multiplier. In particular, there is no abelian neo-difference set of order $n>4$ if $n$ has a divisor $p$ such that $p^{a} \equiv-1(\bmod n-1)$ for some a.

Proof. Clearly -1 would be a multiplier of order 2 , and thus $-1 \equiv \sqrt{n}(\bmod n-1)$ by Theorem 5.4, which is impossible for $n>4$. Then the second assertion follows using Theorem 5.3.

The following simple but important observation was already used by Hughes [12], [13], though the first explicit statement seems to occur in Kantor's paper [17].

Lemma 5.11. If $t_{1}, t_{2}, t_{3}, t_{4}$ are multipliers of an abelian neo-difference set of order $n$ with $t_{1}-t_{2} \equiv t_{3}-t_{4}(\bmod \exp G)$, then $\exp G$ divides the least common multiple of $t_{1}-t_{2}$ and $t_{1}-t_{3}$.

Proof. By Lemma 5.2, we may assume that $D$ is fixed by all numerical multipliers. Therefore $\delta \in D$ implies $t_{i} \delta \in D$ for $i=1, \ldots, 4$. By hypothesis, $t_{1} \delta-t_{2} \delta=t_{3} \delta-t_{4} \delta$ which can only hold if $t_{1} \delta=t_{2} \delta$ or $t_{1} \delta=t_{3} \delta$. Thus the order of every element of $D$ divides the least common multiple of $t_{1}-t_{2}$ and $t_{1}-t_{3}$; as $D$ generates $G$, we obtain the assertion.

Together with Theorem 5.3, this leads to the following result which strengthens the work of Hughes [13, Theorem III.3] and gives strong restrictions on the possible orders of abelian neo-difference sets.

Theorem 5.12. There is no abelian neo-difference set whose order is divisible by any of the following pairs of primes: $(2,3),(2,5),(2,7),(2,11),(2,13),(2,17),(2,19)$, $(2,31),(3,5),(3,7),(3,11),(3,13),(3,17),(3,19),(5,7),(5,11),(5,13),(7,13)$.

Proof. The proof always rests on finding a "repeated difference" and applying Lemma 5.11. We shall illustrate this by considering those cases which are not contained in Hughes' paper. First, let us assume that $n$ is a multiple of $65=5 \cdot 13$; then 5 and 13 and therefore also 25 are multipliers. Now $25-13=13-1=12$, and thus the exponent of $G$ divides 12, by Lemma 5.11. Now Theorem 5.1 implies that $G$ is cyclic, and hence $n-1$ divides 12 , which is absurd. The case where $n$ is a multiple of 91 is handled analogously. Next, assume that $n$ is a multiple of 34 ; then 2 and 17 are multipliers. Now $17-16=2-1=1$, and thus the exponent of $G$ divides 15 , by Lemma 5.11. Note that at least one of the primes 3 and 5 divides $n-1$. As $p=2$ has even order modulo $q$ for both $q=3$ and $q=5$, Theorem 5.7 shows that $n$ has to be a square. By Corollary 5.6, we get the existence of an abelian neo-difference set of order $m=\sqrt{n}$. Clearly, $m$ is again a multiple of 34 , and so this argument may be 
continued ad infinitum, which gives the desired contradiction. The cases where $n$ is a multiple of 38 or 62 are excluded similarly.

The above results have been used together with the aid of a computer to show that every abelian neo-difference set of order $\leqslant 1,000$ has prime power order, see [22]; it should be easy enough to extend this range considerably. Of course, it is conjectured that $n$ is necessarily a prime power. Some further restrictions are due to Tanenbaum [27], Hughes [12] and Pankin [21]. As we do not have new proofs for the results in question, we will not state them here and refer to the original papers instead.

Acknowledgement. This work was partially supported by GNSAGA, by the Italian Ministry for University, Research and Technology (project: Strutture geometriche, combinatoria e loro applicazioni) and the Università di Roma "La Sapienza" (project: Gruppi, Grafi e Geometrie). To a large extent, the research for this paper was done during several visits of the second author to the University of Rome "La Sapienza"; he gratefully acknowledges the hospitality and financial support extended to him. The authors are indebted to Yutaka Hiramine and Bill Kantor for some helpful e-mail exchanges concerning the topic of this paper. They also acknowledge the efforts of an anonymous referee whose suggestions resulted in noticeable cuts to the size of this paper.

\section{References}

[1] A. Barlotti, Le possibili configurazioni del sistema delle coppie punto-retta $(A, a)$ per cui un piano grafico risulta $(A, a)$-transitivo. Boll. Un. Mat. Ital. (3) 12 (1957), 212-226. MR 19,674c Zbl 0077.13802

[2] T. Beth, D. Jungnickel, H. Lenz, Design theory. Cambridge Univ. Press 1999. MR 2000h:05019 Zbl 0945.05004

[3] A. Blokhuis, A. Brouwer, H. Wilbrink, Hermitian unitals are code words. Discrete Math. 97 (1991), 63-68. MR 93b:51017 Zbl 0758.05025

[4] M. J. de Resmini, D. Ghinelli, D. Jungnickel, Arcs and ovals from abelian groups. Des. Codes Cryptogr. 26 (2002), 213-228. MR 1919878 Zbl pre01785710

[5] P. Dembowski, Finite geometries. Springer 1968. MR 38 \#1597 Zbl 0159.50001

[6] P. Dembowski, F. Piper, Quasiregular collineation groups of finite projective planes. Math. Z. 99 (1967), 53-75. MR 35 \#6576 Zbl 0145.41003

[7] D. Ghinelli, D. Jungnickel, Finite projective planes with a large abelian group. In: Surveys in combinatorics. Cambridge Univ. Press 2003.

[8] D. Ghinelli, D. Jungnickel, Piani proiettivi finiti e neo-insiemi di differenze. Quaderni Elettronici del Seminario di Geometria Combinatoria Univ. di Roma "La Sapienza", to appear.

[9] M. Hall, Projective planes. Trans. Amer. Math. Soc. 54 (1943), 229-277. MR 5,72c Zbl 0060.32209

[10] Y. Hiramine, Difference sets relative to disjoint subgroups. J. Combin. Theory Ser. A 88 (1999), 205-216. MR 2000k:05053 Zbl 0938.05015

[11] J. W. P. Hirschfeld, Projective geometries over finite fields. Oxford Univ. Press 1998. MR 99b:51006 Zbl 0899.51002 
[12] D. R. Hughes, Planar division neo-rings. Ph.D. Thesis, University of Wisconsin, Madison 1955.

[13] D. R. Hughes, Planar division neo-rings. Trans. Amer. Math. Soc. 80 (1955), 502-527. MR 17,45lb Zbl 0066.28201

[14] D. R. Hughes, Partial difference sets. Amer. J. Math. 78 (1956), 650-674. MR 18,921b Zbl 0072.38101

[15] D. R. Hughes, F. C. Piper, Projective planes. Springer 1982. MR 48 \#12278 Zbl 0267.50018

[16] D. Jungnickel, K. Vedder, On the geometry of planar difference sets. European J. Combin. 5 (1984), 143-148. MR 85i:05047 Zbl 0543.05010

[17] W. M. Kantor, Projective planes of type I-4. Geom. Dedicata 3 (1974), 335-346. MR 52 \#1114 Zbl 0337.50014

[18] W. M. Kantor, M. D. Pankin, Commutativity in finite planes of type I-4. Arch. Math. (Basel) 23 (1972), 544-547. MR 47 \#9416 Zbl 0248.50026

[19] H. Lenz, Kleiner Desarguesscher Satz und Dualität in projektiven Ebenen. Jber. Deutsch. Math. Verein. 57 (1954), 20-31. MR 15,893c Zbl 0055.13801

[20] L. J. Paige, Neofields. Duke Math. J. 16 (1949), 39-60. MR 10,430b Zbl 0040.30501

[21] M. D. Pankin, On finite planes of type I-4. Ph.D. thesis, University of Illinois at Chicago Circle 1971.

[22] M. D. Pankin, Finite planes of type I-4. In: Proceedings of the International Conference on Projective Planes, 215-218, Washington State Univ. Press, Pullman, Wash. 1973. MR 50 \#14482 Zbl 0271.50023

[23] G. Pickert, Projektive Ebenen. Springer 1955, 1975. MR 17,399e Zbl 0066.38707

[24] T. Ralston, On the embeddability of the complement of a complete triangle in a finite projective plane. Ars Combin. 11 (1981), 271-274. MR 82m:05020 Zbl 0465.05013

[25] B. Segre, Ovals in a finite projective plane. Canad. J. Math. 7 (1955), 414-416. MR $17,72 \mathrm{~g} \mathrm{Zbl} 0065.13402$

[26] A. P. Sprague, Translation nets. Mitt. Math. Sem. Giessen no. 157 (1982), 46-68. MR 84j:05036 Zbl 0499.51002

[27] P. Tannenbaum, On the nonexistence of certain finite projective planes of Lenz-Barlotti type I-4. Boll. Un. Mat. Ital. A (5) 15 (1978), 137-139. MR 58 \#5284 Zbl 0384.51006

[28] J. C. D. S. Yaqub, The Lenz-Barlotti classification. In: Proc. Proj. Geometry Conference. Univ. Illinois, Chicago (1967), 129-162, 1967. Zbl 0176.17703

[29] J. C. D. S. Yaqub, On finite projective planes of Lenz-Barlotti class I-3. In: Number theory and algebra, 349-361, Academic Press 1977. MR 57 \#1269 Zbl 0375.50018

[30] H. Zassenhaus, Über endliche Fastkörper. Abh. Math. Sem. Hamburg 11 (1935), 187-220. Zbl 0011.10302

Received 17 December, 2002; revised 12 February, 2003

D. Ghinelli, Dipartimento di Matematica, Università di Roma "La Sapienza", 2, Piazzale Aldo Moro, 00185 Roma, Italy

Email:dina@mat.uniroma1.it

D. Jungnickel, Lehrstuhl für Diskrete Mathematik, Optimierung und Operations Research, Universität Augsburg, 86135 Augsburg, Germany

Email: jungnickel@math.uni-augsburg.de 